\title{
LA ENSEÑANZA-APRENDIZAJE DE LA LENGUA EXTRANJERA A TRAVÉS DE LA ESTRATEGIA DE LAS WEBQUESTS: UN CASO PRÁCTICO EN EL CAMPUS VIRTUAL DE LA U.C.M.
}

\section{Resumen}

Este artículo presenta un proyecto de Innovación Educativa sobre cómo integrar las TIC en el currículo de la formación inicial del profesorado de lengua extranjera (inglés). El proyecto giró en torno al uso de las WebQuests. Para llevarlo a cabo, se creó un editor de WebQuests y se utilizó la plataforma WebCT de la UCM, así como nuestro sitio Web.

Las WebQuests son actividades útiles para integrar las TIC en la clase de lenguas extranjeras y fomentar el aprendizaje colaborativo. En este estudio se diseñaron e implementaron en el primer curso de Magisterio, dos WebQuests en torno a temas del programa oficial de Lengua Inglesa I. Los resultados muestran cómo las tareas centradas en la lectura, la escucha y la escritura han ayudado al alumnado a desarrollar sus destrezas orales.

Palabras clave: TIC, WebQuest, plataforma WebCT, aprendizaje cooperativo, currículo, lengua Inglesa 


\title{
TEACHING AND LEARNING A FOREIGN LANGUAGE THROUGH THE WEBQUEST STRATEGY: A PRACTICAL CASE IN THE WEBCT PLAT- FORM
}

\begin{abstract}
This article presents an Educational Innovative Project on how to integrate ICT in a preservice English language teacher education curriculum. The project dealt with the use of WebQuests. A WebQuest editor was created and the WebCT platform at Complutense University in Madrid, as well as our own website were used to carry out the different activities.

WebQuests are a useful tool to integrate ICT in the foreign language classroom and promote cooperative learning. Two WebQuests based on some topics in the syllabus of the first year students were designed and implemented. The results showed how reading, listening and written tasks have helped trainees develop their speaking skills.

Key words: ICT, WebQuest, WebCT platform, cooperative learning, curriculum, English language
\end{abstract}

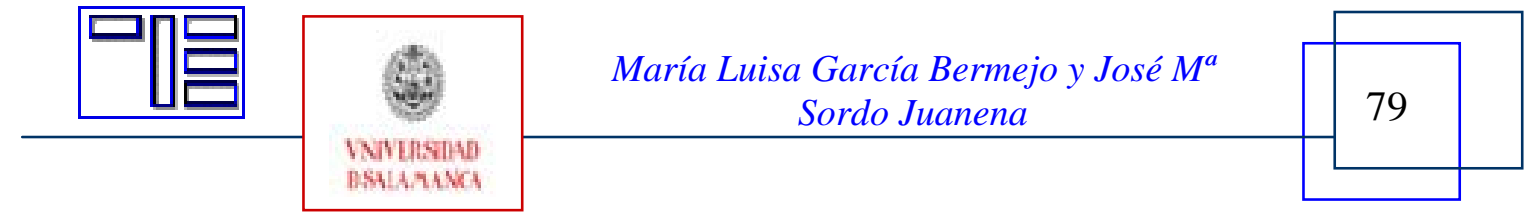




\section{L'ENSEIGNEMENT-APPRENTISSAGE DE LA LANGUE ETRANGERE A TRAVERS LA WEBQUEST : UN CAS PRACTIQUE DANS LA PLATEFORME WEBCT DE L' U.C.M.}

\section{Résumé}

Cet article présente un projet d`innovation éducative pour intégrer les Nouvelles Technologies en classe d'anglais langue étrangère dans la formation initial des professeurs de langues étrangères.

Le projet se fonde sur I` utilisation de WebQuests. On a développé une WebQuest editor et on a utilisé la plateforme WebCT dans I` Université Complutense de Madrid et notre site web aussi. Les activités que proposent les WebQuests sont particulierment idéales pour intégrer les Nouvelles Technologies dans la classe de langue étrangère. Ces activités sont aussi très bien adaptés pour faciliter l’apprentissage coopératif. On a developpé et implémenté deux WebQuests dans le curriculum. Le résultats indiquent que les activités de lecture, écriture et d'audition ont facilité le développement de l'expression orale.

Mots-clés: Technologies de l'Information et de la Communication (TIC), WebQuest, Plateforme WebCT, apprentissage coopératif, currículum, anglais langue étrangère. 


\title{
LA ENSEÑANZA-APRENDIZAJE DE LA LENGUA EXTRANJERA A TRAVÉS DE LA ESTRATEGIA DE LAS WEBQUESTS: UN CASO PRÁCTICO EN EL CAMPUS VIRTUAL DE LA U.C.M.
}

\author{
María Luisa García Bermejo y José Mª Sordo Juanena \\ mlgarber@edu.ucm.es \\ jmsordoj@edu.ucm.es \\ Departamento de Didáctica de la Lengua y la Literatura (Español, Francés e Inglés) y \\ Departamento de Didáctica de las Matemáticas. \\ Facultad de Educación. Universidad Complutense de Madrid
}

\section{1.- INTRODUCCIÓN}

La integración de España en el Espacio Europeo de Educación Superior (EEES) plantea una serie de transformaciones en la organización de las enseñanzas y cambios en los modelos docentes. Así por ejemplo, la metodología docente necesariamente habrá de estar mucho más centrada en el alumno, en su adquisición de competencias y en la creación de conocimiento que en la transmisión del mismo por parte de los docentes. Por otro lado, la implantación del EEES conlleva necesariamente, una mayor implicación del alumno en el proceso de enseñanza-aprendizaje, innovaciones docentes que habrán de incorporar las tecnologías de la información y de la comunicación, recursos económicos y una acción decidida y comprometida de las universidades y el gobierno. En definitiva, un enorme esfuerzo por parte de todos los implicados en el proceso. Los cambios en el sistema de enseñanza han de ser vistos como una oportunidad para reflexionar sobre cómo hemos estado llevando a cabo los procesos de enseñanzaaprendizaje, cómo podemos mejorarlos y qué competencias deberíamos tener los profesores universitarios para afrontar los desafíos del EEES. La adecuación al nuevo modelo sólo será posible si hay una seria implicación en el proceso de todos los miembros de la comunidad universitaria: profesorado, alumnado y personal de administración y servicios y si hay una mejora en las infraestructuras de las universidades para adaptarse a los cambios. Para llevar a cabo los retos que plantea la adaptación al EEES la Universidad Complutense lleva impulsando desde el 2003 una serie de medidas para mejorar la calidad docente, entre ellas, una progresiva inversión en las tecnologías de la información y de la comunicación y sus aplicaciones a la docencia y la investigación y, el impulso del campus virtual, los grupos piloto y los proyectos de Innovación Educativa.

Si bien las TIC no son el fin del EEES, sí es cierto que se convierten en una herramienta clave para el proceso de enseñanza-aprendizaje dentro del EEES. El Espacio Europeo de Educación Superior y en concreto la implantación del crédito europeo, que medirá tanto el trabajo presencial como el no presencial del alumno, abren todo un campo de posibilidades para la enseñanza virtual. La implantación del crédito ECTS supone un cambio importante en cuanto conlleva por un lado, la transformación de los planes de estudio y por otro, un replanteamiento de la metodología a emplear y las estra-

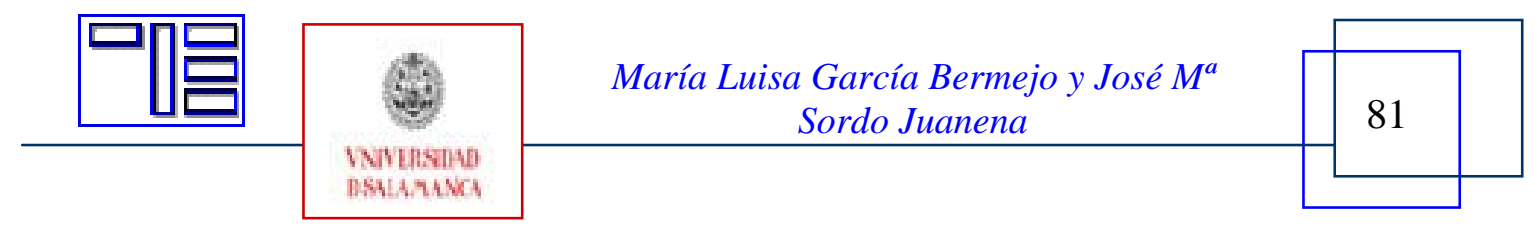


tegias a utilizar para alcanzar los objetivos formativos. Asimismo supondrá un cambio importante con respecto al papel del profesor y del alumno y a los modos de evaluación.

El e-Learning, puede definirse como "el uso de tecnologías basadas en Internet para proporcionar un amplio abanico de soluciones que aúnen adquisición de conocimiento y habilidades o capacidades" (Rosenberg, 2001). Este autor establece unos criterios para considerar una actividad como e-learning: a) que se realice en red, lo que permite una actualización inmediata, almacenaje y recuperación, distribución y poder compartir los contenidos y la información; b) que se haga llegar al usuario final a través de un ordenador utilizando estándares tecnológicos de Internet, y c) que esté centrado en la más amplia visión de soluciones al aprendizaje que vayan más allá de los paradigmas tradicionales de la formación (Rosenberg, 2001, 28-29). El e-Learning irrumpió en el mundo educativo a finales de los años noventa y su progresiva implantación en los diversos niveles educativos, como un modo más de impartir y adquirir conocimiento, está suponiendo un cambio no sólo en el modo de concebir la enseñanza-aprendizaje y los procesos que conlleva, sino también en una visión distinta de cuáles han de ser los nuevos papeles de los profesores y los alumnos dentro de este nuevo paradigma que trata de aunar las nuevas tecnologías con la enseñanza.

Aunque la educación mediante las nuevas tecnologías se lleva aplicando desde hace años en los países mas avanzados tecnológica y económicamente, en concreto más de la mitad de las instituciones universitarias estadounidenses ofrecen alguna forma de educación a distancia por medios electrónicos, en España el proceso está siendo aún lento en parte debido a las carencias tecnológicas de los centros educativos (la inversión necesaria para un cambio tecnológico), la falta de infraestructuras adecuadas y la necesidad real de formar al profesorado en el uso y aplicación efectivos de las nuevas tecnologías.

En términos generales, el e-Learning se viene aplicando en las instituciones educativas bien de forma completa (cursos virtuales, por ejemplo), bien como un complemento a los modos más tradicionales de transmisión del conocimiento. El e-Learning en la educación superior puede constituirse como un complemento más de las formas tradicionales de enseñanza o como el pilar estratégico de la universidad.

La Formación en Red en el contexto de la educación superior y en concreto en las universidades, presenta en la actualidad diversos modelos de implantación del eLearning que van desde virtualidad como complemento a la presencialidad hasta llegar a la virtualidad total. Sangrá (2001) señala diferentes modelos de aplicación del eLearning en las universidades que van ligados a las características de cada universidad, la tecnología seleccionada y las políticas de los diferentes equipos rectorales:

1) Universidad presencial que introduce elementos de virtualidad en su dinámica educativa 2) Universidad presencial con extensión universitaria virtual 3) Universidad virtual adosada a la universidad tradicional 4) Universidad virtual como organización virtual y 5) Espacios virtuales interuniversitarios comunes. La Universidad Complutense es un ejemplo del primer modelo que establece Sangrá: presencial pero con elementos de virtualidad. A través de la plataforma WebCT y de las herramientas que ofrece, el profesor puede colgar contenidos, crear grupos de aprendizaje cooperativo, tutelar a los alumnos etc.

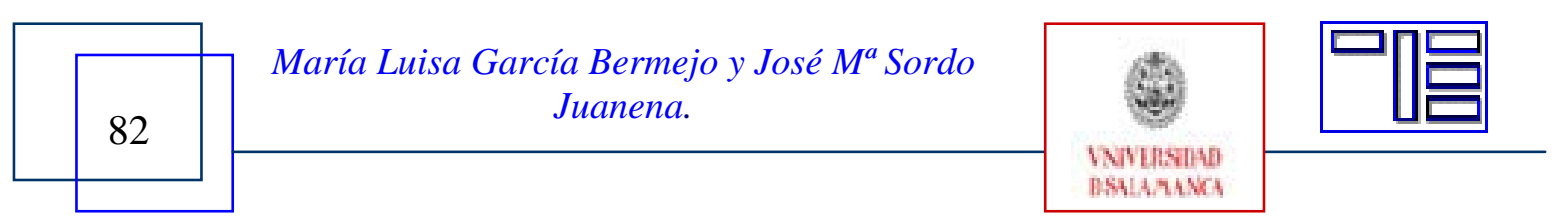


Debido al carácter eminentemente presencial de nuestra universidad, el modelo de aprendizaje por el que hemos apostado hasta el momento en nuestro campo de estudios es el mixto en el que la realización de actividades a través del campus virtual y la Red está unido a las tareas que se hacen en clase.

\section{2.- ELECCIÓN DE UNA METODOLOGÍA DE APRENDIZAJE: LAS WEB- QUESTS Y LA MODALIDAD SEMI-PRESENCIAL}

Con el fin de empezar a abordar los retos derivados de la innovación en las formas de generación y transmisión del conocimiento y hacer frente a los cambios en la docencia que supone la convergencia europea, venimos realizando desde el curso 2004 diversos proyectos de innovación educativa en el marco donde impartimos nuestras clases, la Facultad de Educación y dentro de la titulación de Maestro especialista en Lengua Extranjera.

Las Tecnologías de la Información y de la Comunicación (TIC) han modificado el acceso a la información y al aprendizaje por parte de la sociedad en que vivimos e inciden en el ámbito del conocimiento. La introducción de las TIC en la educación se basa necesariamente en cambios en la metodología y en los contenidos.

En la exposición de motivos de la Ley Orgánica 6/2001, de 21 de diciembre, de Universidades ya se apuntaba la necesidad de una nueva ordenación de la actividad universitaria que permita a las universidades "abordar, en el marco de la información y del conocimiento, los retos derivados de la innovación en las formas de generación y transmisión del conocimiento.” El EEES por otro lado, establece entre otros objetivos la implantación en el 2010 de un sistema de créditos, el modelo ECTS, que por un lado, favorecerá la movilidad de los estudiantes europeos y por otro, supondrá no sólo transformaciones en los planes de estudio sino también cambios metodológicos e innovaciones docentes. El crédito ECTS comportará un nuevo modelo educativo basado en el trabajo del estudiante y no en las horas de clase, es decir, centrado principalmente en el aprendizaje de los estudiantes, no en la docencia de los profesores. La puesta en marcha de los créditos ECTS supondrá por tanto una modificación de las actividades docentes y/o un replanteamiento de las actuales. Las universidades tendrán que establecer diversas metodologías y estrategias para llevar a cabo las diferentes capacidades que la nueva concepción de créditos desarrollará. Igualmente, será necesario replantear diferentes instrumentos de evaluación y autoaprendizaje.

Consideramos que la integración real y el uso de Internet en el currículo puede ser una metodología muy efectiva para llevar a cabo parte de los cambios anteriormente mencionados. Por integrar, entendemos saber utilizar las herramientas y la información que nos ofrece la red en las actividades diarias de la clase, para conseguir los objetivos del currículo y proporcionar oportunidades de aprendizaje a los alumnos. Para ello, llevamos investigando cómo se puede hacer frente a los nuevos retos educativos en la titulación en donde realizamos principalmente nuestra docencia: Maestro especialista en Lengua Extranjera (inglés). Creemos que nuestra propuesta es de especial relevancia por las repercusiones sociales y educativas que necesariamente se deducen de la aplicación de nuevos modos de enseñar y aprender en la formación inicial del profesorado.

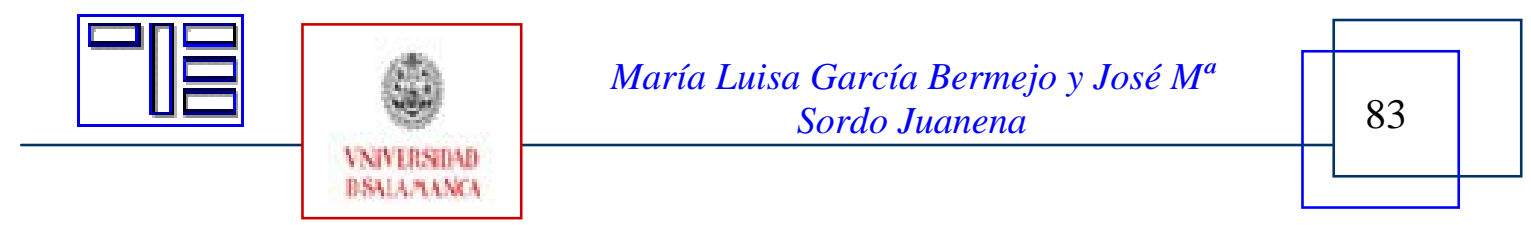


Es necesario adaptar la preparación de los profesores a las demandas del sistema educativo actual español y al del ámbito europeo. El sistema educativo español en todas sus etapas obligatorias señala, entre otros, como uno de los objetivos prioritarios, el conocimiento de varias lenguas extranjeras a la finalización de la etapa escolar y establece que las TIC actuarán como mediadores en el aprendizaje. Para ello, es necesario no sólo desarrollar destrezas básicas en la utilización de las fuentes de información sino también y muy especialmente, aprender a usarlas para encontrar, analizar, intercambiar, transformar y presentar la información y el conocimiento y todo ello, aplicando una metodología que incida en la puesta en práctica de los principios del aprendizaje cooperativo y colaborativo entre los alumnos. Este tipo de aprendizaje hará posible que el conocimiento se construya entre todos y se aprendan a valorar las perspectivas, experiencias y formas de pensar de los demás. Esto es sólo posible si contamos con profesores realmente capacitados en el uso de las nuevas tecnologías en sus diversas áreas de conocimiento, profesores que hayan experimentado este nuevo modo de aprender desde su formación inicial. A menudo, el conocimiento de las Nuevas Tecnologías (NT) y su aplicación para la educación que adquieren nuestros alumnos en su formación inicial se limita a saber buscar información en Internet, utilizar alguna plataforma educativa, conocer programas de software y poco más. Con frecuencia se confunde información con conocimiento. Consideramos que es obligado dar un paso adelante y utilizar las posibilidades que ofrece Internet para ir más allá de una mera búsqueda de información, a menudo realizada en solitario y sin objetivos muy concretos. La tecnología puede y debe ser utilizada para dar la oportunidad a nuestros estudiantes de interactuar socialmente y construir conocimiento. Para ello, a modo experimental, hemos introducido en la asignatura anual de 12 créditos de Lengua Inglesa I (Diplomatura de Magisterio, Especialidad Lengua Extranjera: Inglés) el uso de las nuevas tecnologías a través de la metodología de las WebQuests, ya que se trata de una de las estrategias didácticas más eficaces para incorporar Internet como herramienta educativa y método de trabajo en el aula y además, su incorporación en la clase, hace posible el cambio en los roles que habitualmente profesores y alumnos han tenido. Uno de los fines de nuestro proyecto ha sido investigar cómo a través de tareas realizadas con la ayuda de fuentes en Internet se puede adquirir una lengua extranjera. En nuestro caso queríamos desarrollar WebQuests que tuviesen presente los objetivos lingüísticos y los contenidos temáticos que forman parte de nuestro diseño curricular. Es por ello, que uno de los objetivos principales que hemos tenido presentes a la hora de diseñar e implementar las WebQuests en el aula ha sido el que incluyeran tareas que fomentasen principalmente la adquisición de la lengua extranjera y desarrollasen las destrezas en la L2, además de diversas estrategias y habilidades cognitivas. Partiendo de la lectura y análisis de textos escritos tanto en la red como en el aula, los alumnos en grupos elaboraron una serie de tareas escritas que sirvieron de base para la realización de tareas orales.

El proyecto que realizamos supuso innovaciones en el modo de enseñar y aprender. Creemos que nuestro trabajo benefició no sólo a nuestros alumnos y a todos los componentes del grupo, en cuanto a cambio de actitudes con respecto a los modos de aprender y enseñar, sino que esperamos que los futuros profesores a los que estamos formando, sean capaces de implantar en los centros de enseñanza la metodología que

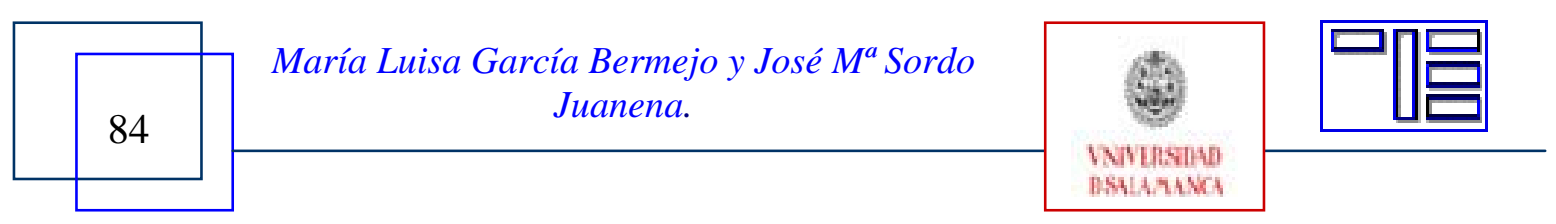


han aprendido. Concebimos nuestro trabajo como un primer peldaño en el andamiaje que necesariamente profesorado y alumnado tendrán que usar para poder cambiar de métodos de enseñanza y actitudes. Es por ello que durante los cursos 2005-2006 y 20062007 hemos seguido creando nuevos contenidos para diversas asignaturas de la especialidad de modo que los alumnos pudieran seguir aprendiendo a través de la metodología propuesta (ver por ejemplo: "Reinventing the classics" , "A healthy life”, Shakespeare and the Elizabethan theatre en http://www.edu-elearning.com/webquests

\subsection{La estrategia didáctica de la WebQuest.}

El concepto de WebQuest fue desarrollado en 1995 por Bernie Dodge, profesor de la Universidad Estatal de San Diego, que junto a su colega Tom March vienen investigando desde hace más de diez años sobre el uso educativo de los recursos que ofrece Internet. Dodge $(1995,1997)$ define el término WebQuest como una actividad orientada a la investigación donde toda o casi toda la información que se utiliza procede de recursos de la Web. Se trata de actividades guiadas y estructuradas, en donde las tareas a realizar están bien definidas y en donde se proporcionan los recursos para poder llevarlas a cabo. De este modo se pretende rentabilizar el tiempo de los estudiantes ya que el alumno se centra más en el uso y la transformación de la información que en su búsqueda. Con ello, se refuerzan y desarrollan los procesos intelectuales en los niveles de análisis, síntesis y evaluación. El modelo de las WebQuests está basado en el aprendizaje cooperativo y en el constructivismo. Todo el proceso se contempla como un andamiaje que posibilita en última instancia la elaboración de un conocimiento que resuelva el problema propuesto por la tarea. Para realizar el producto final, los alumnos trabajan principalmente en grupos y adoptando diferentes roles. Dodge (1995, 1997) y March (1998a, 2001) han definido ampliamente en qué consiste una WebQuest, las partes de que se compone, los principios metodológicos en los que se sustenta este modelo de aprendizaje y las ventajas que ofrece para la educación. Igualmente, han descrito en diversos artículos los pasos a seguir en el proceso de diseñar WebQuests (Dodge, 1998, 2001, 2002b; March, 1998b) así como desarrollado una taxonomía de tareas (Dodge, 2002a) para guiar al docente en el diseño y puesta en práctica de WebQuests.

En el contexto europeo, la línea de investigación sobre el desarrollo y la integración de las WebQuests en un proyecto curricular que nos parece más interesante es la que actualmente están desarrollando los profesores de la Universidad de Utretch Ton Koenraad (2002) y Ton Koenraad y Gerard Westhoff (2003). Ellos proponen un nuevo enfoque en el diseño e implementación de WebQuests que tengan como finalidad última promover un aprendizaje eficaz de una segunda lengua. El concepto de "LanguageQuest” o “TalenQuest”, en holandés, (véase www.talenquest.nl) ideado por estos investigadores, pretende adaptar los principios que subyacen en las WebQuests a los requisitos, basados en las investigaciones sobre la adquisición de segundas lenguas, que necesariamente han de tener estas actividades para promover la adquisición de la lengua.

Toda WebQuest se compone de cinco apartados: Introducción, tarea, proceso y recursos, evaluación y conclusión. A continuación describimos brevemente en qué consisten cada uno de estos apartados:

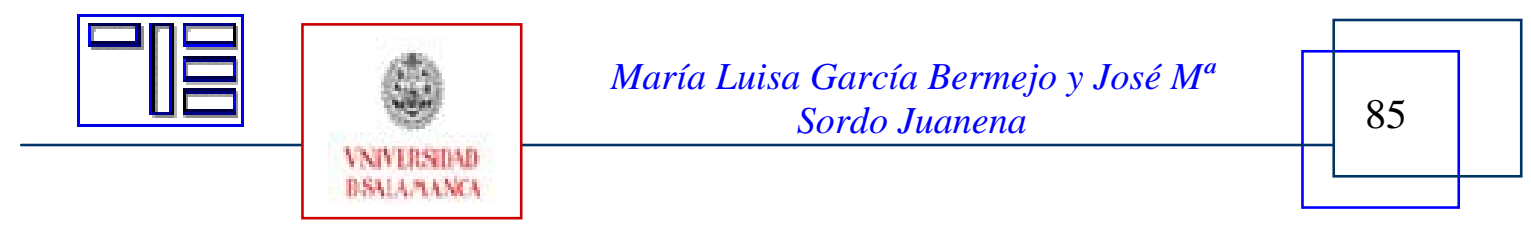


Introducción: Tiene como objetivo presentar el tema motivando al alumnado. Proporciona al estudiante la información sobre lo que se espera de él y prepara el escenario para la acción a realizar. Cuanto más centrado esté el tema en los posibles intereses del alumno y cuanto más lo perciba como relevante mejor.

Tarea: Es el núcleo central de la WebQuest. Define lo que los alumnos deben realizar al final de la actividad. Generalmente han de crear un producto nuevo o resolver un problema y para ello, se requiere que el alumno procese y transforme la información. La tarea se concibe como un trabajo en grupo en donde cada persona asume diversas responsabilidades. De este modo se producen aprendizajes significativos.

Proceso: Describe los distintos pasos que los alumnos deben seguir para realizar la actividad. El proceso además debe contemplar la incorporación de un principio central de las WebQuests: el aprendizaje cooperativo. La WebQuest es una estrategia didáctica en la línea de los principios que ven el conocimiento como algo socialmente distribuido, algo que se construye colectivamente. Es por ello que las propuestas del proceso han de desencadenar actividades de aprendizaje cooperativo.

Recursos: Las fuentes de información, fundamentalmente páginas Web y otros recursos electrónicos a los que el alumno debe acudir para realizar la tarea y cada una de las actividades o subtareas propuestas. Los recursos son seleccionados por el profesor.

La evaluación: Señala lo que se espera de los alumnos y tiene que ser coherente con la tarea. Se verá por tanto si los alumnos han sido capaces de realizar sus tareas, responder a las preguntas formuladas en ellas, fundamentando esas respuestas, haciendo argumentaciones buenas. También se evaluará el producto confeccionado por los alumnos y la calidad del mismo.

La conclusión: Revisa y reactiva los conocimientos adquiridos, anima a continuar el aprendizaje. Es una oportunidad para reflexionar acerca del proceso vivido y sobre lo aprendido.

Para la aplicación de WebQuests en el currículo consideramos que hay que tener en cuenta una serie de características implícitas en esta forma de enseñar y aprender:

- Metodológicas:

La estrategia de la WebQuest da especial importancia a los procesos de aprendizaje y a las dinámicas de grupo. Por un lado, la realización de tareas auténticas y significativas que conlleva la WebQuest fomenta un aprendizaje centrado en el alumno y basado en desarrollar, entre otras, sus habilidades de síntesis, análisis, comprensión, transformación del conocimiento así como la resolución de problemas. Por otro lado, promueve el aprendizaje cooperativo y colaborativo, haciendo posible diferentes roles de colaboración entre los alumnos, fomentando tanto el trabajo individual como en grupo para construir el conocimiento. Finalmente, en el área de lengua, permite integrar las cuatro destrezas de una forma natural, siendo especialmente un instrumento de comprensión lectora activa y selectiva en el que la lectura es medio y no fin.

- Papel del profesor:

El profesor es el encargado de proporcionar a los grupos de clase con WebQuests de interés relacionadas con la programación de la asignatura que les motiven. Para la confección de la WebQuest, selecciona sitios en la red que sean de

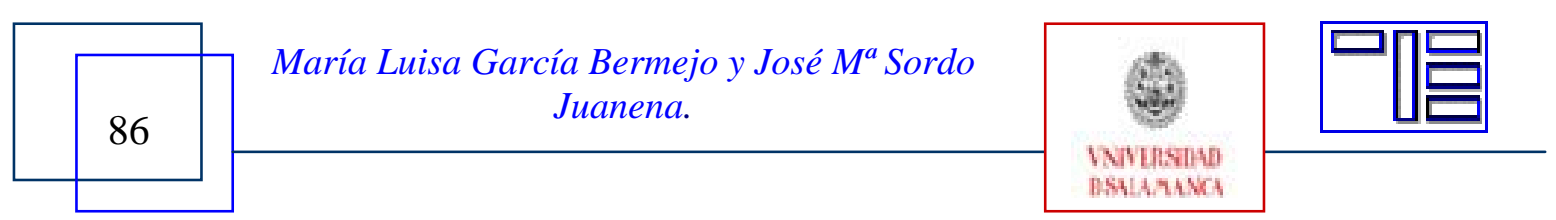




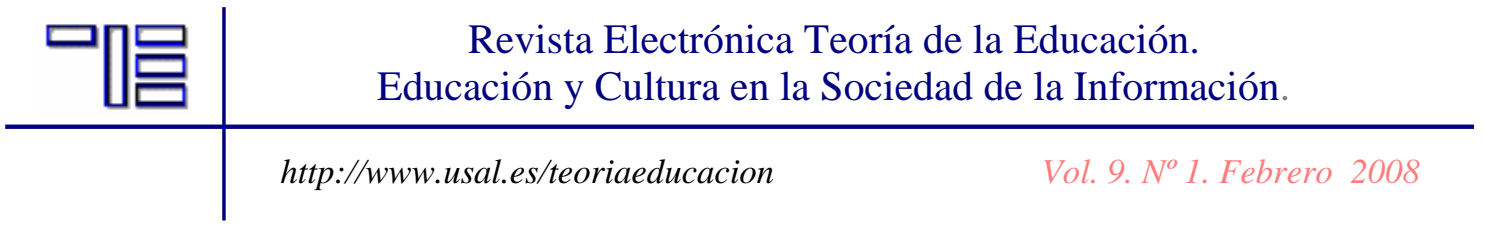

utilidad a sus alumnos para poder realizar las tareas que se les proponen. Es por lo tanto principal responsable de proporcionar los recursos para que la elaboración del producto final por parte de los alumnos sea posible. También es el organizador del entorno cooperativo y de las diferentes tareas que los alumnos han de llevar a cabo. Suministra a los distintos miembros de cada grupo con diversos roles y les asesora y orienta acerca de cómo llevar a cabo la tarea propuesta. Debe de ser claro a la hora de comunicar lo que se espera de los estudiantes y cómo serán evaluados.

- Papel de los alumnos:

La WebQuest, al aplicar los principios del aprendizaje cooperativo, fomenta la toma de responsabilidad de los alumnos en su propio aprendizaje y el del grupo, ya que cada miembro es responsable individualmente con respecto al logro del objetivo común y la construcción del conocimiento compartido. Para Dodge una buena WebQuest es la que crea situaciones que obligan al alumnado a depender unos de otros. Igualmente, la estrategia de la WebQuest promueve el concepto de "aprender a aprender."

\section{3.- UN CASO PRÁCTICO EN UNA CLASE DE FORMACIÓN DE PROFESO- RES}

En este trabajo presentamos una experiencia didáctica con alumnos de primer curso de la especialidad de Magisterio, Lengua Extranjera. Durante el curso académico 20042005 se diseñaron y se pusieron en práctica tareas en la red (WebQuests) centradas en desarrollar las cuatro destrezas (leer, oír, hablar y escribir), en un segundo idioma, con el fin de la integrar las TIC en una asignatura del currículo: Lengua Inglesa I. En concreto, se diseñaron e implementaron dos Webquests sobre temas del programa oficial. Partiendo de la lectura y análisis de textos escritos tanto en la red como en el aula, los alumnos en grupos elaboraron una serie de tareas escritas que sirvieron de base para la realización de tareas orales. Para llevar a cabo esta experiencia, se creó un editor de WebQuests, se utilizó la plataforma WebCT de la UCM y nuestro servidor (http://www.edu-elearning.com). Ello permitió que las aportaciones de los alumnos quedasen registradas y que los alumnos implicados en este proyecto pudiesen establecer contacto entre ellos vía correo electrónico, foro y chat, así como enviar sus trabajos al profesor. Las WebQuests diseñadas "Job opportunities abroad” y "Madrid in English" pueden verse en http://www.edu-elearning.com/webquests (invitado). Por otro lado, se incluyó como parte del trabajo un Weblog o cuaderno de bitácoras, en donde nuestros alumnos hacían sus reflexiones sobre todo el proceso. Ello nos permitió detectar problemas y evaluar su grado de satisfacción con respecto a esta forma de trabajo. Hay que tener presente que carecían de experiencia en WebQuests y ésta era la primera vez que realizaban tareas en la red de forma colaborativa .

La experiencia, que tuvo lugar dentro de un Proyecto de Innovación Educativa del Vicerrectorado de Innovación y Espacio Europeo de Educación Superior tenía tres grandes objetivos:

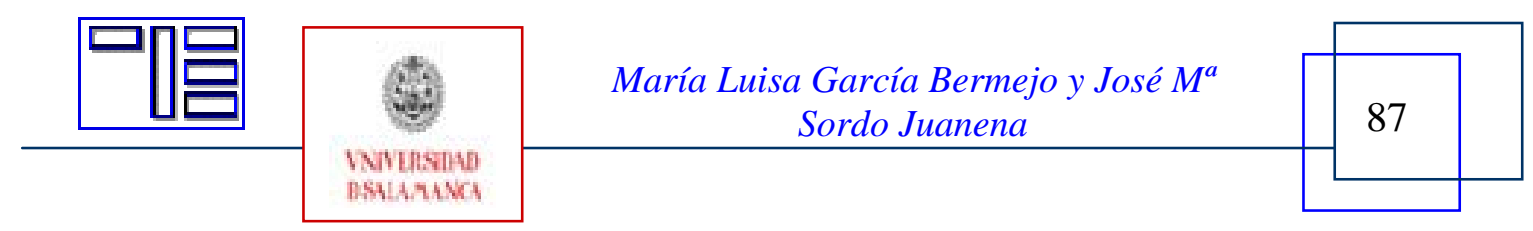


Revista Electrónica Teoría de la Educación.

Educación y Cultura en la Sociedad de la Información.

http://www.usal.es/teoriaeducacion

Vol. 9. No 1. Febrero 2008

- Usar e integrar Internet en el aula, para trabajar de modo cooperativo, con el fin de que los futuros maestros puedan aplicar durante su vida profesional, la metodología y las estrategias didácticas a las que han sido expuestos durante su formación.

- Diseñar, desarrollar e implementar WebQuests por parte de los miembros del equipo investigador y en última instancia, por parte de los futuros profesores en formación.

- Diseñar, desarrollar e implementar un editor de WebQuests. Incorporar la plataforma virtual WebCT a nuestra docencia y diseñar un sitio Web donde cualquier alumno o profesor que se registre pueda editar sus páginas Web y además puedan ser usadas por cualquier otro alumno o profesor.

Para realizar un seguimiento del proyecto se utilizaron diversos métodos: cuestionarios, observación directa, grado de consecución de los objetivos planteados en las tareas realizadas, cuaderno de bitácoras etc.

Dado que en la realización de este proyecto se entrecruzaban la creación de contenidos, la pedagogía, la tecnología y la organización, nos pareció de suma importancia contar con un equipo investigador interdisciplinar. Ello supuso una profunda colaboración e interdependencia entre los participantes dado que, por un lado, no solo se trataba de crear contenidos para la clase (WebQuests en nuestro caso), sino también implementarlas en la red a través de la plataforma WebCT y el sitio Web creado. Igualmente, había que aprender a integrar las TIC en la clase y trabajar con los alumnos con el material diseñado, la plataforma WebCT y distintas formas de comunicación en la Red ( Weblog,....) y las herramientas telemáticas elegidas para desarrollar la experiencia (WebCT, Weblog etc).

Para poner en práctica nuestro proyecto elegimos un modelo educativo mixto o "blended-teaching" en el que las tareas para la red diseñadas e implementadas estaban íntimamente relacionadas con las actividades que se realizaban en la clase presencial, siendo ésta una ayuda o complemento esencial para poder completar las actividades en Red. Dichas actividades giraban en torno al currículum y a aspectos literarios, lingüísticos, culturales e históricos relacionados con el mundo anglosajón. Como bien señalan los expertos de la UOC María Teresa Arbués y Lluís Tarín (Pelegrín, 2007: 206, 211) los materiales didácticos son fundamentales dentro de la enseñanza virtual. Para estos autores, "En el contexto de la formación virtual, la creación, diseño y estructuración de los materiales y recursos didácticos son etapas de un proceso fundamental que se ha de abordar con rigor para asegurar la calidad de los programas y la facilitación del aprendizaje”. Para ellos, además "los contenidos se diseñan al servicio de las personas que aprenden”. Fue teniendo en cuenta estos aspectos que elaboramos dos WebQuests que resultasen atractivas y de utilidad para nuestros alumnos.

Dada la inexperiencia de nuestros alumnos en el uso educativo de las TIC y en trabajar con WebQuests, nos pareció apropiado comenzar nuestro proyecto realizando una identificación y análisis de necesidades. Para ello, se confeccionó un cuestionario sobre nivel de conocimientos lingüísticos y tecnológicos de los alumnos y actitudes y creencias con respecto a Internet y su uso educativo. Igualmente se preguntó a los alumnos sobre conocimientos previos en el uso de Internet y las TIC en general. Una

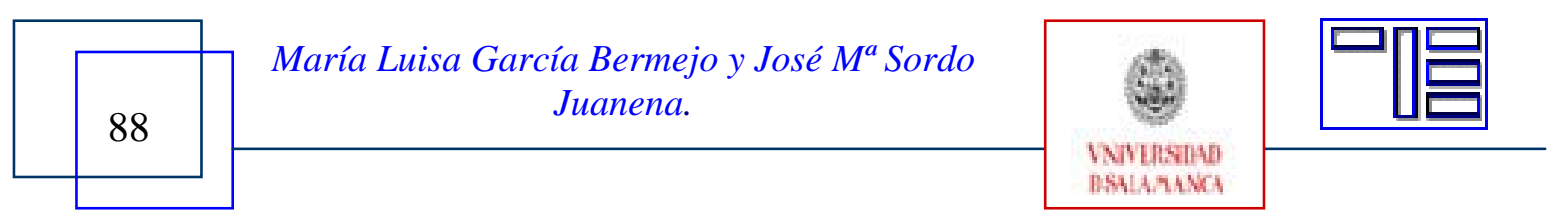


vez conocidas las necesidades de nuestros alumnos y puesta en marcha la parte técnica, se dedicaron varias sesiones a familiarizarles con las TIC en general y la metodología de la WebQuest, en particular, Para ello, la clase hizo una WebQuest de prueba: "Today's news" diseñada por la profesora Isabel Pérez (www.isabelperez.com). Durante la realización de esta primera WebQuest, se detectaron problemas de organización, se valoró el proceso y se pensó en posibles soluciones para el futuro y la implantación y desarrollo de la WebQuest a realizar por el equipo investigador. A partir de esa información y tras la fase de prueba, se diseñaron e implementaron dos WebQuests: Job opportunities abroad y Madrid in English: Film Review. En ambas, los alumnos trabajaron en sus proyectos en el horario lectivo y también fuera de clase, como parte de los créditos prácticos que tienen contemplados en la asignatura de Lengua Inglesa I (ocho créditos teóricos y cuatro prácticos). Puesto que en nuestro caso las WebQuests han de tener entre sus fines la mejora del nivel lingüístico de nuestros alumnos, las WebQuests diseñadas incluían tareas en torno a las cuatro destrezas: leer, escribir, hablar y escuchar. En una primera fase que iba de la lectura a la escritura. Los alumnos en pequeños grupos, partiendo de la lectura y análisis de textos escritos en soporte informático, y con el apoyo también de actividades en el aula en soporte papel donde se trabajó el léxico específico para incrementar la base léxica y otros aspectos relevantes, elaboraron sus tareas escritas (e.g. un folleto informativo, una reseña de una película). En esta primera fase de escritura, cada alumno primero individualmente y luego en pequeño grupo confeccionó diversos borradores que leían tanto el profesor como los compañeros. En este proceso, se utilizaron distintas técnicas de corrección: en parejas y por parte del profesor. Pretendíamos desarrollar en nuestros alumnos el sentido de "audiencia” ya que como señala Hayland (2002), "el profesorado juega un papel fundamental a la hora de responder a la producción escrita de los alumnos, pero también se puede entrenar a los compañeros para que respondan y comenten trabajos de otros”. De esta manera los alumnos escribían con un propósito determinado, concibiendo la escritura como un acto social donde se negociaban significados para conseguir un fin común. Una vez recogidas las sugerencias de los compañeros y el profesor, cada grupo enviaba su versión final de la tarea al profesor. El producto final de los alumnos se componía de textos multimodales al añadir un soporte visual. En una segunda fase, que iba de la escritura a la producción oral, el producto escrito final servía de base para la realización de tareas orales (presentación en clase del producto final) que se grabaron en vídeo para poder ofrecer retroalimentación a cada grupo. Finalmente, toda la clase junto al profesor ofrecía sugerencias a las presentaciones realizadas y tenía una conversación informal sobre la experiencia en la realización de las WebQuests.

\section{4.- DIFICULTADES Y LIMITACIONES EN LA IMPLANTACIÓN DE LA ME- TODOLOGÍA EN EL CAMPUS VIRTUAL}

La puesta en marcha de la estrategia metodológica de las WebQuests ha supuesto un cambio de mentalidad en la manera de pensar y trabajar tanto por parte del profesor como de los alumnos. Por un lado, el profesor tiene que acostumbrarse a dejar su papel de único trasmisor de conocimiento para convertirse en facilitador y orientador de las tareas que él mismo ha propuesto a sus alumnos basándose en sus intereses y en las

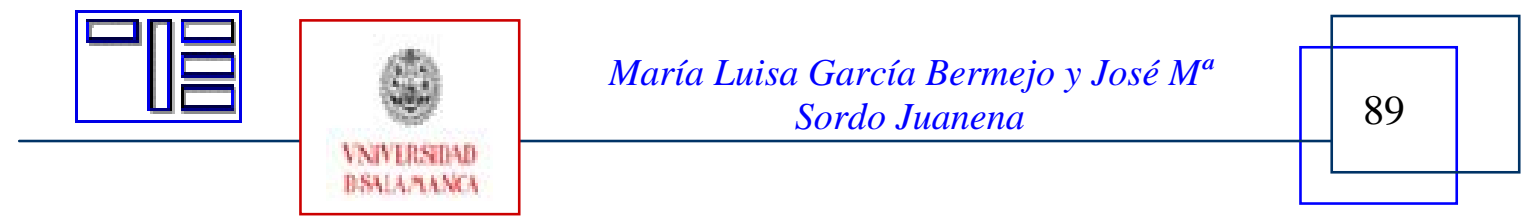


competencias que se espera adquieran a través de las tareas encomendadas. Por otro lado, el alumno se convierte en protagonista activo de su aprendizaje y el de sus compañeros, asume una mayor responsabilidad en su propio aprendizaje, aprende a aprender y a construir conocimiento compartido.

El aprendizaje mixto (blended learning) choca a veces con dificultades de tipo organizativo. La mala organización espacio-temporal de nuestras facultades, la todavía escasa dotación informática en nuestras aulas, que a menudo conlleva problemas de navegación lenta en nuestras aulas de informática o escasa facilidad de acceso a Internet por parte de nuestros alumnos crea a menudo dificultades a nuestros alumnos para trabajar con Internet. A esto hay que añadir las ausencias y bajas de los alumnos que a menudo entorpecen el trabajo en equipo, o los diversos niveles tecnológicos y lingüísticos que los estudiantes poseen.

\section{5.- CONCLUSIONES}

Como muy bien se aprecia en los cuestionarios inicial y final (véase http://www.edu-elearning.com/director/proyecto.htm ) que se les pasó a los alumnos, así como en los diferentes trabajos realizados por ellos y en la grabación y observación de clases por parte del equipo investigador, los alumnos, desde el punto de vista de adquisición de conocimientos lingüísticos y tecnológicos, mejoraron sensiblemente ambos campos al tener que manejarse en la red para realizar las tareas lingüísticas propuestas. En especial se comprobó a través de la observación directa y de grabaciones en video y audio, cómo el proceso de lectura, escritura y refuerzo en clase del léxico específico tiene como principal consecuencia la mejora de la producción oral de los alumnos. Con respecto a los procedimientos para aprender, la experiencia sirvió para desarrollar en ellos la cooperación y la responsabilidad individual y de grupo, al habérseles propuesto tareas que necesariamente implicaban el trabajar con otros compañeros y llegar a acuerdos. El hecho de trabajar en grupo sirvió para mitigar los problemas propios de alumnos con distintas habilidades lingüísticas y fomentar las relaciones sociales del grupo. Además, en lo que se refiere a objetivos actitudinales, la metodología empleada supuso un incremento notable de la motivación tanto por el método de trabajo como por la utilidad de las actividades propuestas. Hemos de señalar que los alumnos perdieron el miedo a usar la tecnología para aprender, pudieron desarrollar su creatividad en la realización de las tareas y aprendieron a trabajar en grupo y colaborar entre sí. De hecho, uno de los beneficios del proyecto ha sido la mayor cohesión entre los alumnos gracias a tener que interaccionar forzosamente. Por último, las WebQuests han resultado ser un instrumento eficaz para integrar las nuevas formas de comunicación con la enseñanza y el aprendizaje, utilizando nuevas estrategias didácticas y pedagógicas

El proyecto de innovación, su diseño y desarrollo junto con los contenidos elaborados por el equipo investigador durante el curso académico 2004-2005 (dos WebQuest diseñadas e implementadas y los aspectos tecnológicos) así como ejemplos de los trabajos realizados por los alumnos a través de la WebCT y nuestro sitio Web, pueden verse en el siguiente enlace:

http://www.edu-elearning.com/director/proyecto.htm

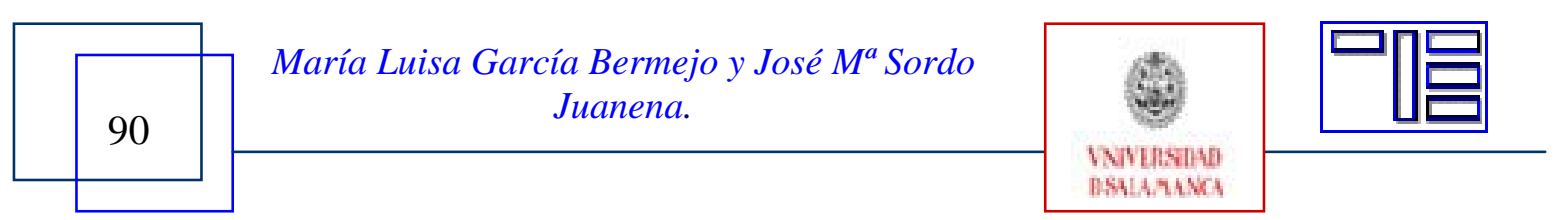




\section{6.- REFERENCIAS.}

DODGE, B. (1995): WebQuests: A technique for Internet-based learning. Distance Educator 1 Vol. (2), pp.10-13. (1997) (1995): Some thoughts about WebQuests http://edweb.sdsu.edu/courses/EdTec596/About_WebQuests.html (1 de marzo 2004) (1998): Building blocks of a WebQuest.

http://edweb.sdsu.edu/people/bdodge/webquest/buildingblocks.html (2 septiembre 2001)

(2001): "Five rules for writing a great webquest", Learning and leading with technology, May 2001.

(2002a): WebQuest taskonomy: a taxonomy of tasks http://webquest.sdsu.edu/taskonomy.html (1 marzo 2004) (2002b): WebQuest design process.

http://webquest.sdsu.edu/webquest/designsteps/index.html ( 1 de marzo 2004)

KOENRAAD, A. L.M. (2002): TalenQuest: WebQuests for Modern Languages. In J. Colpaert \& W. Decoo \& M. Simons \& Sv. Bueren (Eds.), CALL professionals and the future of CALL research, Proceedings CALL 2002 (pp.159-168). Antwerp: University of Antwerp. Available at: http://www.koenraad.info/CALL

KOENRAAD, A. L.M. y WESTHOFF, G.J. (2003): Can you tell a Language Quest when you see one? Design criteria for TalenQuests. Paper presented at the 2003 Conference of the European Association for Computer Assisted Language Learning. EUROCALL 2003, University of Limerick.

HAYLAND, K. (2002). Teaching and Researching Writing. Essex: Longman

MARCH, T. (1998a): Why WebQuests?, an introduction http://www.ozline.com/webquests/intro.html (31 de mayo de 2001) (1998b):

The Webquest Design Process http://www.ozline.com/webquests/design.html (1 de abril 2004) (2001): Working the Web for Education http://www.ozline.com/learning/theory.html (31 de mayo 2001). PELEGRÍN, C. (coord.) (2007). e-Learning: las mejores prácticas en España. EDIPE. ROSENBERG, M.J. (2001): E-learning. Strategies for Delivering Knowledge in the Digital Age. New York: McGraw-Hill.

SANGRÁ, A. (marzo 2001): La calidad en las experiencias virtuales de Educación Superior, revista Cuadernos IRC ,5.

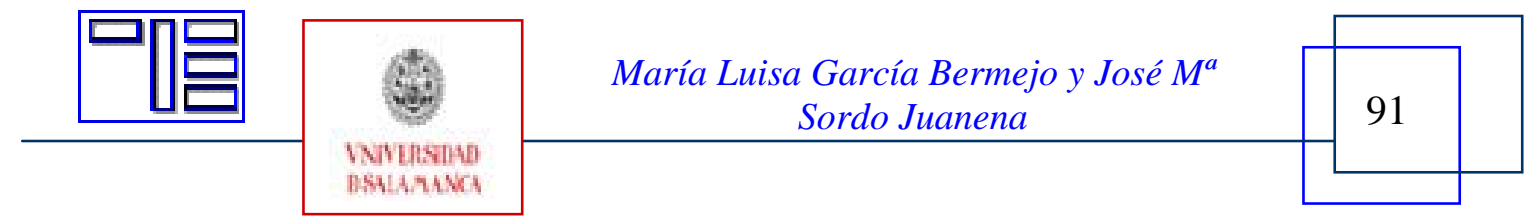

